
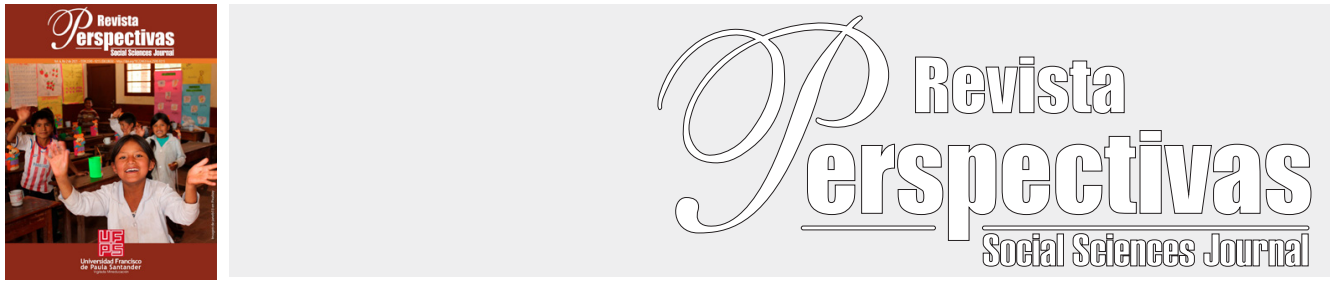

Artículo Original

https://doi.org/10.22463/25909215.2922

\title{
Que Comunicar No nos Cueste la Vida
}

That Communicating doesn't Cost Us Our Lives

Laura Nataly Galvis-Velandia ${ }^{1}$, Jessica Ortega ${ }^{2}$, Natalia Duque ${ }^{3}$

${ }^{I}$ Magister En Educación, profesor, docente investigador de la Universidad Francisco de Paula Santander, miembro de los Grupos de investigación GICOM / GITS. lauranatalygv@ufps.edu.co,Orcid:0000-0003-2444-8398.Cúcuta, Colombia

${ }^{2}$ Magister en comunicación Digital de, Comunicadora Social (Universidad Francisco de Paula Santander) redes@gmail.com. Cúcuta, Colombia

${ }^{3}$ Periodista, (Universidad de Antioquia) redaccion@colombiainforma.info, agencia de comunicación popular Colombia Informa. Cúcuta, Colombia.

Como Citar: L.N. Galvis-Velandia, J. Ortega, N. Duque, “Que Comunicar No nos Cueste la Vida.”. Perspectivas, vol. 6, no. 2, pp. 47-52, 2021.

Recibido:February 02, 2021; Aprobado: April 05, 2021.

\section{RESUMEN}

\begin{tabular}{|c|c|}
\hline $\begin{array}{l}\text { Comunicar, } \\
\text { Contextos De Riesgo, } \\
\text { Periodistas, } \\
\text { Censura, } \\
\text { Autocensura. }\end{array}$ & $\begin{array}{l}\text { El presente artículo, parte de los resultados de la investigación titulada "Comunicación en contextos de riesgo: } \\
\text { un estudio de caso de los periodistas de la agencia de comunicación popular Colombia Informa" realizado por } \\
\text { el grupo de investigación de comunicación y medios (GICOM) a la agencia de comunicación popular Colombia } \\
\text { Informa, donde se realizo un abordaje desde lo conceptual frente a la comunicación, el periodismo, la censura } \\
\text { y autocensura. Adicionalmente se analizaron los aspectos teóricos y conceptuales a la luz de la metodología } \\
\text { cualitativa con un estudio fenomenológico desde el contexto actual, dando como resultado aspectos relevantes } \\
\text { frente a la autoprotección y el reto de la autocensura, concluyendo en la importancia de la protección y no } \\
\text { vulneración de los derechos humanos aquellas personas que día a día luchan por informar la verdad. }\end{array}$ \\
\hline & ABSTRACT \\
\hline $\begin{array}{l}\text { Keywords: } \\
\text { Communicate, } \\
\text { Risk Contexts, } \\
\text { Journalists, } \\
\text { Censorship, } \\
\text { Self-Censorship. }\end{array}$ & $\begin{array}{l}\text { This article, part of the results of the research entitled "Communication in risk contexts: a case study of } \\
\text { journalists from the popular communication agency Colombia Informa" carried out by the communication and } \\
\text { media research group (GICOM) to the popular communication agency Colombia Informa, where a conceptual } \\
\text { approach was made to communication, journalism, censorship and self-censorship. Additionally, the theoretical } \\
\text { and conceptual aspects were analyzed in the light of the qualitative methodology with a phenomenological } \\
\text { study from the current context, resulting in relevant aspects regarding self-protection and the challenge of self- } \\
\text { censorship, concluding on the importance of protection and non-violation of human rights those people who } \\
\text { day by day fight to inform the truth. }\end{array}$ \\
\hline
\end{tabular}

\section{Introduction}

En Colombia ejercer el periodismo o la comunicación popular es caminar con la esperanza de construir narrativas junto a la gente que lucha, pero con la posibilidad de ser perseguida, detenida o golpeada en el camino. Aunque las leyes consignan el derecho a informar y ser informado, la realidad cuenta con un repertorio de acciones que obstaculizan esta labor y que configuran constantes escenarios de riesgo para quienes la desempeñan. El presente artículo, realizado por el grupo de investigación de comunicación y medios (GICOM) a la agencia de comunicación popular Colombia Informa; destaca que la libertad de expresión, el derecho a informar y recibir información, así como de fundar medios masivos de comunicación, son algunos de los elementos que hacen parte de la Constitución Política de 1991, carta magna que debería materializarse en los derechos y deberes que tienen todos los ciudadanos en el país. Sin embargo, los ataques contra medios de comunicación independientes y alternativos, así como la violencia dirigida contra la prensa han pintado un panorama oscuro pero real que debe ser analizado (CONSTITUCIÓN). 


\section{Materiales y Métodos}

El desarrollo metodológico se dio a la luz del paradigma cualitativo con un estudio fenomenológico desde el contexto actual que permitió comprender los fenómenos sociales explorados desde las perspectivas de las diferentes poblaciones en su vida cotidiana. El enfoque de investigación empleado por los presentes autores para el análisis del estado actual de la libertad de prensa en Colombia, se enfoca en el estudio de caso de los periodistas de la agencia de comunicación popular Colombia Informa, además de la revisión bibliográfica y estado del arte de la realidad del periodismo en el país; utilizando para ello fuentes bibliográficas indexadas, artículos periodísticos, reseñas históricas, noticias, entre otros; con el objetivo de estudiar cualitativamente las condiciones actuales, ya que este enfoque, según (Gonzales 2013), permite estudiar un fenómeno a partir de los procesos sociales, considerando las características de los actores, y cómo esto conduce el actuar de los individuos inmersos en la situación estudiada.

\section{Resultados y Discusión}

Estado del Arte: Al analizar el estado del arte de la investigación de la que se desprende este capítulo se identificó que nada más en los últimos cuatro años han sido asesinados ocho periodistas y se han denunciado 618 amenazas, según datos de la Fundación para la Libertad de Prensa (Flip). Estas cifras ya de por sí escandalosas, excluyen el terrorismo de Estado evidenciado en el mes de mayo del 2021 en diferentes ciudades de Colombia y que ha afectado especialmente a quienes desempeñan esta labor. Los principales victimarios han sido las fuerzas del Estado entre policía y ejército, así como grandes conglomerados empresariales que, además de ser dueños de más del $50 \%$ de los medios de comunicación colombianos, también han forjado estrechos vínculos con los gobiernos de turno hasta el punto de camuflarse en el senado y el gabinete según manifiestan los informantes claves del estudio de caso.
A las amenazas y las agresiones físicas se suma la estrategia de perfilamientos que fue denunciada al comienzo de 2020, a través de la cual se hizo seguimiento y se aplicó la inteligencia militar a varios periodistas críticos con el Gobierno. Incluso un descuido en Twitter dejó al descubierto una lista creada por la cuenta delEjército Nacional deColombia llamada "Oposición". Estas prácticas generaron zozobra e indignación entre periodistas preocupados por su integridad física al ser considerados como el enemigo por parte de unas fuerzas militares que están adoctrinadas para eliminar lo distinto. La Agencia de Comunicación de los Pueblos Colombia Informa hizo parte de esta lista según lo manifestado en la entrevista.

A las amenazas y las agresiones físicas se suma la estrategia de perfilamientos que fue denunciada al comienzo de 2020, a través de la cual se hizo seguimiento y se aplicó la inteligencia militar a varios periodistas críticos con el Gobierno. Incluso un descuido en Twitter dejó al descubierto una lista creada por la cuenta del Ejército Nacional de Colombia llamada “Oposición”. Estas prácticas generaron zozobra e indignación entre periodistas preocupados por su integridad física al ser considerados como el enemigo por parte de unas fuerzas militares que están adoctrinadas para eliminar lo distinto. La Agencia de Comunicación de los Pueblos Colombia Informa hizo parte de esta lista según lo manifestado en la entrevista. En las demás regiones no fue distinto. Mientras en las ciudades fueron golpeados y retenidos periodistas, en el campo además fueron asesinados. Esto ocurrió con Abelardo Liz, un comunicador indígena del Norte del Cauca quien el 13 de agosto se encontraba en una jornada de Liberación de la Madre Tierra cuando el ejército abrió fuego. Abelardo grabó el momento exacto en el que las balas lo impactaron. (Colombia Informa. 17 de agosto de 2020. [Crónica] Las balas del Ejército contra un comunicador y un liberador Nasa. https://www.colombiainforma.info/cronicalas-balas-del-ejercito-contra-un-comunicador-y-unliberador-nasa) 
Este año el panorama no cambia. El 2021 trajo consigo el retorno de un movimiento popular fortalecido en las calles que ha sido brutalmente reprimido por las fuerzas estatales, dejando hasta el momento un saldo de al menos 490 personas heridas y 52 personas asesinadas entre el 28 de abril y el 10 de mayo, según cifras de la Campaña Defender la Libertad: un asunto de todas. Esta violencia contra la movilización ha sido legitimada a través de los medios de comunicación corporativos que estigmatizan la protesta social. (Campaña Defender la Libertad, un asunto de todas. 11 de mayo de 2020. Boletín informativo \#10: \#ParoNacional. https:// defenderlalibertad.com/boletin-informativo-10paronacional)

Periodistas y comunicadoras populares han estado durante dos semanas acompañando a la ciudadanía indignada que decretó Paro Nacional desde el 28 de abril. En estos quince días de jornadas se ha evidenciado un panorama de agresiones a la prensa sin precedentes, asegura la Flip, quienes además registran 124 agresiones en este periodo de tiempo; es decir, un aproximado de ocho casos por día.

Uno de ellos ocurrió el 10 de mayo en medio de las manifestaciones en Bogotá, cuando un corresponsal de Colombia Informa se encontraba transmitiendo en vivo la represión por parte del Escuadrón Móvil Antidisturbios -Esmad- contra los manifestantes. En plena transmisión se logra ver cómo la tanqueta se ubica justo al frente de un grupo de personas identificadas como prensa entre las que se encontraba el corresponsal. Una vez allí, uno de los uniformados se asoma por la ventana y lanza un gas directamente hacia el periodista, impactando en su pierna derecha.

"Puede verse cómo el disparo se hace de forma directa y no cumple con la normativa que exige la utilización de este tipo de artefactos (que deben lanzarse de forma parabólica). De igual manera, podemos ver cómo la tanqueta se detiene y los uniformados tienen tiempo suficiente para -desde el interior del vehículo- identificar al personal de prensa", narra el corresponsal. (Campaña Defender la Libertad, un asunto de todas. 11 de mayo de 2020. Boletín informativo \#10: \#ParoNacional. https:// defenderlalibertad.com/boletin-informativo-10paronacional)

A estas agresiones físicas se suma la censura de la que han sido víctimas los medios de comunicación alternativos que registran la violencia en las calles. El bloqueo y la eliminación de publicaciones en las redes sociales, la interferencia en la señal de quienes transmiten en vivo, las bodegas de boots que denuncian cuentas y contenidos relacionados con el Paro Nacional, son algunas de las situaciones que se han presentado y que han dificultado el trabajo de quienes están informando. Pero ni siquiera con esta batalla desproporcional y frontal contra los medios y periodistas se ha dejado de informar. (Galvis L, Ortega J, Duque N (2021) "Comunicación en contextos de riesgo: un estudio de caso de los periodistas de la agencia de comunicación popular Colombia Informa" Universidad Francisco de Paula Santander. Colombia)

Estudio de caso: Así mismo dentro de las entrevistas se resalta el sentir frente a que las consecuencias han sido devastadoras para un país que se hace llamar "la democracia más antigua de América Latina". Por un lado, la posibilidad de construir otros espacios de comunicación que logren incidir en la opinión pública es completamente remota. Los medios corporativos se han asegurado de tener el monopolio de la información, generando un cerco mediático a través del cual solo se proyecta públicamente lo que es conveniente para los mismos. Así, se tiene la perspectiva de que solo existe una única narrativa de los hechos, mientras que la gente pelea con uñas para exponer posturas diversas, realidades distintas, lo que en últimas se llama contra informar. Dentro de las categorías que emergieron se encuentra la de "La lucha contra informativa" que, según lo plasmado en los resultados de la investigación, ha tomado relevancia en los últimos años para los movimientos populares 
en el país. Así, las poblaciones históricamente excluidas y empobrecidas han construido iniciativas que alimentan un abanico importante de medios independientes, alternativos y populares. Su objetivo ha sido romper con el cerco y posicionar realidades distintas que escapan de lo que es conveniente para la hegemonía y su estatus quo.

Otra categoría que emerge es la de "poder a través de la violencia" en donde refieren que ante un Estado que mantiene la concentración de poder a través de la violencia, exponer la verdad se ha configurado como un acto de valor y rebeldía. Han sido las periodistas y comunicadores populares quienes han captado en terreno las violaciones de Derechos Humanos que son cometidas constantemente por las fuerzas estatales y paraestatales, así como quienes han comenzado a exponer las telarañas de corrupción que han sostenido al gobierno hasta la actualidad. Asimismo, han sido los periodistas y comunicadores populares quienes han expuesto su cuerpo y mente a las violencias que el mismo Estado ha impulsado para atemorizar, detener y silenciar la verdad y la indignación cuando esta se comienza a asomar. Estas prácticas han configurado un escenario sumamente riesgoso para la libertad de expresión y la prensa alternativa; "la violencia contra la prensa ocurre con la misma sistematicidad y permisividad como sucedía en décadas pasadas, durante los años más oscuros de Colombia”, asegura la Flip en su revista publicada en febrero de este año donde exponen cuál ha sido el estado de la libertad de expresión en el 2020 .

\section{Discusión: La Autoprotección y el Reto de la Autocensura}

Al realizar el proceso de triangulación de la información socavada a lo largo de la investigación surge un análisis frente al concepto de autoprotección y autocensura. Ya que a este escenario de agresiones sistemáticas contra la prensa también se suma la "autocensura", como una actitud de silencio que contribuye a la consolidación del cerco informativo. Esto puede ocurrir por motivos estructurales que responden a una marginalidad selectiva por parte de los espacios en los que se replica la información. (Galvis L, Ortega J, Duque N (2021) “Comunicación en contextos de riesgo: un estudio de caso de los periodistas de la agencia de comunicación popular Colombia Informa" Universidad Francisco de Paula Santander. Colombia)

Por lo anterior es pertinente aclara que se entiende por censura que " es el conjunto de actuaciones del Estado, grupos de hecho o de existencia formal capaces de imponer a un manuscrito o a las galeradas de la obra de un escritor - con anterioridad a su publicación - supresiones o modificaciones de todo género, contra la voluntad o el beneplácito del autor" (M. L. ABELLÁN, (1982) Censura Y Autocensura En La Producción Literaria Española, Nuevo Hispanismo, No 1, 1982, pp. 169-180) y por autocensura como "las medidas previsoras que, consciente o inconscientemente, un escritor adopta con el propósito de eludir la eventual reacción o repulsa que su texto pueda provocar en todos o algunos de los grupos o cuerpos del Estado facultados para imponerle supresiones o modificaciones con su consentimiento o sin él" (M. L. ABELLÁN, Censura y creación literaria en España (1939-196) (Barcelona, Ediciones Península, 1980), páginas 1)

Por ejemplo, en Colombia rara vez se conoce lo que ocurre en la región suroriental del país. Allí viven comunidades campesinas, indígenas, alejadas de los centros urbanos y de los escenarios de producción y distribución de bienes y servicios. Ellas no figuran en la agenda de los medios corporativos y pocas veces lo hacen en la de los medios alternativos, según lo manifiestan las periodistas de Colombia informa.

Adicionalmente refieren que otro de los motivos por los que se presenta la autocensura tiene que ver con el contexto en el que se ejerce y las garantías de seguridad que brinda el lugar. Así, en varias regiones del país, periodistas y comunicadoras saben que sus palabras se pueden devolver en balas, por lo que 
muchos de ellos terminan migrando de su territorio o incluso exiliados en el exterior.

El Artículo 12 de la Ley 51 de 1975 en Colombia signa que "Los funcionarios públicos y especialmente las autoridades de policía, garantizarán la libre movilización del periodista y su acceso a los lugares de información, para el pleno cumplimiento de su misión informativa, salvo en casos reservados conforme a las leyes. La violación de lo dispuesto anteriormente será causal de mala conducta, sancionable con destitución".

Esta ley hace parte de la infraestructura en términos de derechos que debería sostener el Estado colombiano pero que en lo real solo representa el fantasma de lo que debería ser. "En este escenario los espacios de comunicación y periodismo alternativo han optado por aprender e implementar medidas de autocuidado que les permitan ejercer su labor minimizando los riesgos. Estas van desde el uso de gafas, cascos y máscaras de gas, hasta la construcción de protocolos prácticos a la hora de estar en terreno, y técnicas de cuidado digital." (Galvis L, Ortega J, Duque N (2021) "Comunicación en contextos de riesgo: un estudio de caso de los periodistas de la agencia de comunicación popular Colombia Informa" Universidad Francisco de Paula Santander. Colombia).

\section{Conclusiones}

Es importante aclarar que las estrategias de autocensura surgen porque las garantías para informar son prácticamente nulas y el Estado no expresa ningún interés en que esto cambie.

Mientras tanto, el país continúa en convulsión y quienes informan continúan en las calles y los espacios de redacción pensando en estrategias para que construir la memoria de nuestra resistencia para que "comunicar no nos cueste la vida"

Todo lo anterior permite concluir que la censura es uno de los grandes retos para los medios alternativos que disputan ideas y agendas con la hegemonía. Lograr la articulación y la construcción de escenarios propios de comunicación es vital para quienes consideran que la comunicación es un derecho inalienable de todos los pueblos. La comunicación y el periodismo independiente, alternativo y popular son indispensables para darle sentido a lo que ocurre y formar una opinión crítica, incluso si constantemente insisten en exterminarla.

\section{Referencias}

Agencia de Comunicación de los Pueblos Colombia Informa. (2014). Manual de estilo. Colombia.

Aharonian, A. (2017). El asesinato de la verdad. La Fogata Editorial. Colombia.

Campaña Defender la Libertad, un asunto de todas. 11 de mayo de 2020. Boletín informativo \#10: \#ParoNacional. https://defenderlalibertad.com/ boletin-informativo-10-paronacional/

Colombia Informa. 10 de mayo de 2020. Policía agrede libertad de prensa en desalojos de Ciudad Bolívar. https://www.colombiainforma.info/ policia-agrede-libertad-de-prensa-en-desalojosde-ciudad-bolivar/

Colombia Informa. 17 de agosto de 2020. [Crónica] Las balas del Ejército contra un comunicador y un liberador Nasa. https://www.colombiainforma. info/cronica-las-balas-del-ejercito-contra-uncomunicador-y-un-liberador-nasa/

Constitución Política de Colombia [Const]. Art. 20. 7 de julio de 1991 (Colombia).

Fundación para la Libertad de Prensa [Flip]. 22 de julio de 2020. Perfilamientos ilegales a periodistas y defensores de derechos humanos en la mira de los EE.UU. https://flip.org.co/ index.php/es/informacion/pronunciamientos/ item/2548-perfilamientos-ilegales-a-periodistasy-defensores-de-derechos-humanos-en-la-mira- 
de-los-ee-uu

Fundación para la Libertad de Prensa [Flip]. 23 de noviembre de 2020. Estigmatizaciones irresponsables contra periodistas que cubren manifestaciones ponen en riesgo a los reporteros. https://flip.org.co/index.php/es/informacion/ pronunciamientos/item/2626-estigmatizacionesirresponsables-contra-periodistas-que-cubrenmanifestaciones-ponen-en-riesgo-a-losreporteros

Fundación para la Libertad de Prensa [Flip]. 8 de febrero de 2021. El periodismo no es el enemigo. https://flip.org.co/index.php/en/publicaciones/ informes-anuales/item/2666-el-periodismo-noes-el-enemigo

Fundación para la Libertad de Prensa [Flip]. 11 de mayo 2021. Silencio de autoridades en ataques contra el periodismo promueve la censura. https://flip.org.co/index.php/es/informacion/ pronunciamientos/item/2721-silencio-deautoridades-en-ataques-contra-el-periodismopromueve-la-censura

Fundación para la Libertad de Prensa [@FLIP org]. (13 de mayo de 2021). En 15 días de manifestaciones sociales, hemos evidenciado un panorama de violencia contra la prensa sin antecedentes. Twitter. https://witter.com/FLIP org/status/1392919836213317637

Galvis L, Ortega J, Duque N (2021) “Comunicación en contextos de riesgo: un estudio de caso de los periodistas de la agencia de comunicación popular Colombia Informa" Universidad Francisco de Paula Santander. Colombia

MCJ Marquez, LNG Velandia, MAG Castellanos (2019) El dilema de la guerra o la paz: representaciones sociales sobre la paz de jóvenes del colegio San José de Cúcuta, Revista Perspectivas 4 (2), 31-38.
M. L. ABELLÁN, Censura y creación literaria en España (1939-196) (Barcelona, Ediciones Península, 1980), páginas 9, 87 y sigs)

((M. L. ABELLÁN, (1982) Censura Y Autocensura En La Producción Literaria Española, Nuevo Hispanismo, $\mathrm{N}^{\mathrm{o}} 1,1982$, pp. 169-180)

Reporteros sin Fronteras., Federación Colombiana de Periodistas [Fecolper]. 2015. ¿A quién pertenecen nuestros medios? http://www. monitoreodemedios.co/

Zárate, F. (2018). La autocensura periodística: una opción de vida y muerte. Universidad de Manizales. Colombia. 